\title{
Travail non libre et rapports sociaux de sexe - À propos des programmes canadiens d'immigration temporaire
}

\author{
Elsa Galerand et Martin Gallié*
}

\section{Résumé}

L'analyse proposée ici défend la nécessité d’opérer un déplacement vis-à-vis des termes qui semblent dominer pour l'heure la réflexion sur les nouvelles formes d'esclavage ou de servitude pour y intégrer pleinement les rapports sociaux de sexe en tant que rapports sociaux de production et d'exploitation. Dans cette perspective, à partir de deux études sur le terrain réalisées auprès des travailleuses domestiques et des travailleurs agricoles soumis aux programmes canadiens d'immigration, il sagit de chercher à voir comment les rapports de travail qui sont rassemblés sous les concepts « d'esclavage moderne » et de « travail non libre » produisent non seulement une classe de travailleurs exclue du salariat canonique, mais aussi et simultanément une main-d’œuvre racisée, intrinsèquement sexuée : des «non-ressortissants » qui sont aussi des hommes et des femmes dont les corps sont différemment mis au travail, exploités et marqués.

Mots clés : Rapports sociaux de sexe, travail non libre, travailleuses domestiques, travailleurs agricoles, migrants, exploitation, esclavage

\begin{abstract}
The analysis proposed here defends the necessity to operate a shift in the terms that seem to dominate, at present, the thinking on new forms of slavery or servitude, in order to fully integrate gender relations as production and exploitation social relationships. In this regard, based on two field studies performed with domestic and agricultural workers subjected to the Canadian immigration programs, we must try to see how the work relations amalgamated under the concepts of "modern slavery" and "unfree labour" give rise not only to a working class that is excluded from the canonical wage-earning class, but also and simultaneously to a racialized, intrinsically sexual workforce: "non-nationals" who are also male workers and female workers whose bodies are put to work, exploited and branded differently.
\end{abstract}

Key words: gender relations, unfree labour, domestic workers, agricultural workers, migrants, exploitation, slavery

\footnotetext{
* Nous tenons à remercier les évaluateurs anonymes ainsi qu'Adelle Blackett pour ses précieux commentaires sur des versions préliminaires de ce texte. Cette recherche a bénéficié du soutien financier du Conseil de recherches en sciences humaines du Canada (CRSH).
}

Canadian Journal of Law and Society / Revue Canadienne Droit et Société, 2018, Volume 33, no. 2, pp. 223-241. doi:10.1017/cls.2018.20 


\section{Introduction}

Au Canada, des formes de travail «non libre " ${ }^{1}$ sont notamment observées dans les deux secteurs du travail migrant temporaire le plus déqualifié : le travail agricole saisonnier et le travail domestique. Les recherches qui s'y intéressent montrent en effet que les programmes canadiens d'immigration de travail temporaire sont, dans ces deux secteurs, dérogatoires au droit commun en ce qu'ils autorisent et organisent des formes « exceptionnelles » d'exploitation. Ils privent les travailleuses et travailleurs du droit de se syndiquer, des régimes de protection sociale et de la liberté de circuler. Ils attachent les personnes concernées à un secteur d'emploi comme à un employeur ou une employeuse unique ${ }^{2}$.

Ces programmes produisent ainsi un travail " d'immigré » ${ }^{3}$ et une fraction de classe singulièrement exploitée : des travailleurs racisés, assujettis à un régime spécial qui leur est réservé et qui consiste précisément à les priver des droits du travail rattachés à la citoyenneté. Et cette fraction de classe est aussi intrinsèquement sexuée : divisée en hommes et femmes dont les corps sont différemment mis au travail et différemment exploités. C’est sur ce clivage interne au travail non libre, qui procède d'une division sociale du travail et qui produit des rapports différenciés au travail, aux espaces de travail comme au temps travaillé et, en bout de course, aux conditions de lutte, que nous proposons de réfléchir.

Pour préciser les enjeux de la démarche adoptée ici, nous reviendrons dans un premier temps sur quelques-uns des débats qui traversent actuellement la réflexion sur le travail non libre. Il s'agira de mettre en discussion deux corpus de théories critiques qui ont en commun de prendre directement appui sur l'héritage marxien. Le premier se situe du côté des critiques marxistes de l'économie politique pour lesquelles les mouvements de prolétarisation, déprolétarisation, reprolétarisation ${ }^{4} \mathrm{du}$ travail font partie de la dynamique même du rapport capital/travail. De ce point de vue, le travail non libre constitue donc un enjeu crucial et actuel de la lutte des classes et non plus une forme résiduelle de mise au travail. Le second corpus se situe du côté des critiques féministes matérialistes et imbricationnistes de l'économie politique.

1 Dans lesquelles le travailleur est privé « de la liberté élémentaire de vendre librement sa seule capacité de travail au plus offrant comme un salarié bénéficiant du droit du travail "normal" sans être assujetti au "régime spécial" réservé aux étrangers ", selon la définition de Yann MoulierBoutang, «Formes de travail non libre. "Accumulation primitive : préhistoire ou histoire continuée du capitalisme?” " Cahiers détudes africaines 179-180 (2005) : 1069-1092, à la p. 1077.

2 Vic Satzewich, Racism and the Incorporation of Foreign Labour: Farm Labour Migration to Canada since 1945 (London: Routledge, 1991); Tanya Basok, Tortillas and Tomatoes: Transmigrant Mexican Harvesters in Canada (Montréal: McGill-Queen's University Press, 2002); Leah Vosko, Precarious Employment: Understanding Labour Market Insecurity in Canada (Montréal, Kingston: McGill-Queen's University Press, 2006); Judy Fudge et Fiona MacPhail, « The Temporary Foreign Worker Program in Canada: Low-Skilled Workers as an Extreme Form of Flexible Labor ", Comparative Labor Law and Policy Journal 31, $\mathrm{n}^{\circ} 5$ (2009) : 101-139; Fay Faraday, Made in Canada: How the Law Constructs Migrant Workers' Insecurity (Toronto: Metcalf Foundation, 2012); Adrian A. Smith, "Troubling "Project Canada": the Caribbean and the making of "unfree migrant labor" " Canadian Journal of Latin American and Caribbean Studies 40, $\mathrm{n}^{\circ} 2$ (2015) : 274-293; Aziz Choudry et A. Smith Adrian, Unfree Labour? Struggles of Migrant and Immigrant Workers in Canada (Oakland: PM Press, 2016).

3 Abdelmalek Sayad, L'immigration ou les paradoxes de l'altérité (Paris : Raisons d'agir, 2006).

4 Tom Brass, Labour Regime Change in the Twenty-First Century - Unfreedom, Capitalism and Primitive Accumulation (Leiden - Boston: Brill, 2011). 
Les rapports sociaux de sexe et de race y sont envisagés en tant qu'ils constituent des régimes spécifiques d'exploitation ou d'appropriation du travail par lesquels se produisent des hommes et des femmes, des ressortissants et des non-ressortissants, des racisés/racisées et des non-racisés/-racisées. Dans cette perspective, les rapports sociaux de sexe, de race et de classe sont irréductibles les uns aux autres. Les relations complexes qu'ils entretiennent aux autres sont cependant constitutives des transformations du travail, des rapports de force en présence et des conflits sociaux.

C'est donc à partir de ce cadre théorique que nous proposons d'examiner, dans un second temps, les programmes canadiens de migration de travail temporaire. Nous verrons que le travail migrant temporaire " a deux sexes " ${ }^{5}$, et ce sont les implications de cette sexuation dans et par le travail que nous tenterons d'illustrer à l'aide des résultats issus de deux enquêtes sur le terrain.

La première concerne les travailleuses domestiques soumises au Programme des aides familiaux (PAF). Elle a été conduite en partenariat, dans le cadre du Service aux collectivités de l'UQAM, avec les militantes de Pinay qui travaillent activement pour la défense des droits des travailleuses domestiques philippines au Québec, depuis 1991. L'enquête consistait à documenter les conditions de vie, de travail et de lutte des aides familiales résidantes. Dans ce contexte, outre les réunions préparatoires à la construction du projet de recherche en lui-même avec les représentantes de Pinay, deux ateliers de travail avec 8 employées de maison ont été organisés. Par la suite, 33 travailleuses ont été rencontrées individuellement. Cette enquête a été menée entre juin 2012 et février $2014^{6}$.

La seconde enquête portait sur les travailleurs agricoles saisonniers employés au Québec dans le cadre du Programme des travailleurs agricoles saisonniers (PTAS) ou du volet agricole du Programme des travailleurs étrangers temporaires (PTET). La recherche a profité de la collaboration du syndicat des Travailleurs et travailleuses unis de l'alimentation et du commerce (TUAC) de Saint-Rémi et de celle de deux militants qui y étaient engagés. Avec eux, nous avons élaboré un guide d'entretien dirigé, et organisé des rencontres avec les travailleurs au Centre d'appui. En octobre 2014, nous avons mené une première série d’entretiens avec 51 travailleurs, puis une seconde avec 42 travailleurs, à l'été 2015. Ils étaient mexicains, guatémaltèques ou honduriens ${ }^{7}$.

5 Nous reprenons ici la formule de Danièle Kergoat et Helena Hirata, « La classe ouvrière a deux sexes ", Politis 4 (1993) : 55-58.

6 Au moment de l'enquête, les travailleuses étaient dans l'obligation de résider chez les employeurs/ employeuses. Depuis 2014, cette obligation a été abrogée par le gouvernement fédéral. La résidence n'est donc plus obligatoire de jure, elle est désormais laissée au « libre-choix » des parties au contrat. Les effets concrets de cette réforme ne sont pas documentés à ce jour. Elsa Galerand, Martin Gallié et Jeanne Ollivier-Gobeil, Travail domestique et exploitation : le cas des travailleuses domestiques philippines au Canada (PAFR), Rapport de Recherche SAC-PINAY, Labour Law and Development Research Laboratory, McGill, janvier 2015, <http://www.mcgill.ca/lldrl/files/ 1ldrl/15.01.09_rapport_fr_vu2.5.1_1.pdf>.

7 Martin Gallié, Jeanne Ollivier-Gobeil et Caroline Brodeur, La néo-féodalisation du droit du travail agricole : Étude de cas sur les conditions de travail et de vie des travailleurs migrants à Saint-Rémi (Québec), Cahiers du GIREPS nº 8, 2017. <http://www.gireps.org/wp-content/uploads/2017/08/ Rapport_DroitTravail_Online.pdf>. 


\section{Le travail non libre : enjeu de définition et débats théoriques}

Deux types de corpus s'emparent centralement de la question du travail non libre. Ils participent tous deux de la théorie critique mais sont rarement mis en discussion en dépit d'un intérêt convergent pour la critique de l'économie politique.

Le premier renvoie aux théorisations marxistes du " travail non libre " et il existe, à l'intérieur même de ce corpus, des manières divergentes d'envisager son statut et son importance du point de vue de la lutte des classes (1.1). À la " thèse semi-féodale ", selon laquelle le travail non libre est une forme résiduelle précapitaliste et donc secondaire, s'oppose celle de la " prolétarisation/déprolétarisation » du travail en tant que dynamique centrale du conflit de classes.

Cette thèse rejoint pour partie les critiques féministes matérialistes et imbricationnistes de l'économie politique. Celles-ci opèrent cependant une rupture vis-àvis des théories marxistes en contestant l'usage réservé du concept d’exploitation à sa seule forme capitaliste qui exclut de sa définition les divisions sexuelles et raciales du travail. Par opposition, elles mettent en évidence l'existence de différents rapports sociaux de production qui se recomposent et se réorganisent mutuellement et réciproquement, ce qui vient complexifier l'analyse des conflits du travail (1.2).

\subsection{Le travail non libre dans la critique marxiste de l'économie politique}

Comme le rappelle Tom Brass, la question du travail non libre est loin d'être nouvelle pour les théoriciens marxistes et non marxistes de l'économie politique ${ }^{8}$. Depuis le $\mathrm{XIX}^{\mathrm{e}}$ siècle, les libéraux soutiennent que le capitalisme - fonctionnant à plein (fully-functioning) ${ }^{9}$ - est incompatible avec le travail non libre (esclavage, servage, servitude pour dette, indenture, péonage, etc.). Celui-ci ne serait pas suffisamment productif; il ne permettrait pas de produire du travail qualifié; il coûterait finalement trop cher; il bloquerait l'expansion du marché et ne garantirait pas la disponibilité de la main-d'œuvre à long terme. Constituant un frein à l'accumulation capitaliste, il serait finalement voué à disparaître au profit du travail libre. Cette thèse de l'incompatibilité du travail non libre au capitalisme n'est cependant pas propre aux théories libérales. Nombre de théoriciens marxistes défendent également, depuis le XIX ${ }^{\mathrm{e}}$ siècle, que les formes observables de travail non libre sont des survivances semi-féodales (the semi-feudal thesis) ${ }^{10}$, nécessairement temporaires, périphériques ou résiduelles, ou encore quelles constituent les "formes extrêmes " d'un " capitalisme sauvage ». En dépit de ce qui peut les opposer aux théories libérales, ces conceptions marxistes s'accordent en somme sur l'idée que le développement du capitalisme doit conduire - à plus ou moins long terme - à l'éradication des formes de travail non libre. Leur persistance ne saurait être, le cas échéant, que « résiduelle ». L’existence du travail non libre, malgré son ampleur et sa permanence,

8 Tom Brass, Labour Regime Change in the Twenty-First Century - Unfreedom, Capitalism and Primitive Accumulation, (2011), à la p. 2.

9 Tom Brass, «Unfree Labour as Primitive Accumulation? », Capital \& Class 35, n 1 (2011) : 23-38, à la p. 26.

10 Tom Brass, Labour Regime Change in the Twenty-First Century - Unfreedom, Capitalism and Primitive Accumulation (2011), à la p. 25. 
se trouve donc reléguée au rang des problèmes secondaires dans cette analyse puisque "qui se soucie d'une forme résiduelle? »" comme le fait remarquer Christine Delphy dans son plaidoyer pour une théorie générale de l'exploitation; nous y reviendrons.

Si donc la question du statut du travail non libre n'est pas une question nouvelle, elle semble susciter un intérêt renouvelé depuis le milieu des années 1990, où l'OIT (Organisation internationale du travail) et le BIT (Bureau international du travail) ont multiplié les rapports sur « l'esclavage moderne » et "le travail forcé » ${ }^{12}$. Ces rapports rendent compte de la diversité des formes de travail non libre et de son ampleur dans différents secteurs : agriculture, travail domestique, prostitution, travail des enfants ${ }^{13}$. Ils montrent également que, loin d'être cantonné dans ladite " périphérie », il touche au contraire les pays du Sud comme ceux du Nord ${ }^{14}$.

La permanence du travail non libre en système capitaliste est donc aujourd'hui officiellement et empiriquement établie. Les formes qu'il peut prendre font l'objet d'inventaires et de typologies, tandis que les mesures en faveur du « travail décent » visant à les combattre sont inscrites à l'agenda de l'OIT. C'est dans ce contexte qu'il faut situer le " renouvellement " ${ }^{15}$ actuel des débats et des questionnements :

Ces formes de travail non libre sont-elles des "vestiges anachroniques d'un passé féodal ou des "sociétés traditionnelles", constituent-elles un mode de fonctionnement "normal" d'un capitalisme par ailleurs parfaitement inscrit dans la modernité? Peut-on parler d'esclavage moderne? Si nous avons affaire à des formes non libres de travail, comment peuvent-elles subsister dans un système où le «travail libre » est dominant? Accumulation primitive, préhistoire du capitalisme qui ne fait pas partie de son histoire propre, ou bien partie intégrante de l'histoire du "capitalisme historique"? "

Alors que le BIT et l'OIT mobilisent les concepts « d'esclavage moderne » et de " travail forcé » pour désigner ce à quoi s'oppose le " travail décent », plusieurs chercheurs pointent les impasses auxquelles peut conduire le recours à ces deux notions. L'intérêt de réactiver la catégorie d’esclavage ${ }^{17}$ avec « sa qualification de

11 Christine Delphy, « Pour une théorie générale de l'exploitation. Deuxième partie : repartir du bon pied ", Mouvements 1, $\mathrm{n}^{\circ} 31$ (2004) : 97-106, à la p. 97.

12 Roger Botte, "Les habits neufs de l'esclavage ", Cahiers détudes africaines 179-180 (2005) : $651-666$.

13 OIT, Global Estimates of Modern Slavery: Forced labour and forced mariage (Genève, Publication OIT, 2017).

14 Tom Brass, Labour Regime Change in the Twenty-First Century - Unfreedom, Capitalism and Primitive Accumulation (2011), à la p. 25.

15 Stephanie Barrientos, Uma Kothari, Nicola Phillips, "Dynamics of Unfree labour in the Contemporary Global Economy " The Journal of Development Studies 49, $n^{\circ} 8$ (2013) : 1037-1041, à la p. 1037.

16 Yann Moulier-Boutang, «Formes de travail non libre. "Accumulation primitive : préhistoire ou histoire continuée du capitalisme?" " (2005), à la p. 1069.

17 Pour une analyse des débats en cours entre une définition « maximaliste » de «l'esclavage moderne » (défendue par exemple par Kevin Bales) ou une définition « minimaliste » (défendue davantage par Jean Allain et Robin Hickey), nous renvoyons à Chantal Thomas, «Immigration Controls and "Modern-Day Slavery" ", Cornell Law School research paper No.13-86, aux p. 20-26. Du côté de l'anthropologie et la sociologie, voir Alexis Martig et Francine Saillant, "Présentation : L'esclavage moderne : une question anthropologique? », Anthropologie et Sociétés 41, nº 1 (2017) : 9-27. 
moderne ${ }^{18}$ est d'abord mis en cause, et la discussion sur ce point s'organise autour de deux séries de questions : dans quelle mesure le recours à cette " métaphore de l'inacceptable » ${ }^{19}$ est-elle utile du point de vue des luttes? Dans quelle mesure rend-elle compte des transformations en cours, de leur spécificité? Pour Christophe Bormans, par exemple, l'usage de cette notion d'esclavage moderne est problématique dans la mesure où il revient à rejeter « dans le non-capitalisme les formes de mise au travail les plus dégradantes pour lêtre humain ${ }^{20}$.

Alain Morice critique la construction d'un « esclavage métaphorique » dans lequel on tend à regrouper «pêle-mêle un ensemble hétéroclite de rapports d'oppression et de mise au travail basés sur la contrainte et la menace ${ }^{21}$. À partir du cas du « saisonnage ", il avance que l'emploi de la terminologie de l'esclavage est non seulement contestable mais contreproductif sur le plan politique. Parce que l'esclavage désigne, selon lui, « un mode d'appropriation pérenne de personnes considérées comme des choses et constitutif de classes sociales spécifiques ", son usage conduirait à manquer l'une des spécificités du travail saisonnier, à savoir que, en dépit « de la brutalité des rapports sociaux qui le caractérisent parfois, il a les attributs formels de la liberté, parvient au miracle de faire croire au libre choix de ceux qu'il soumet ${ }^{22}$.

La portée de la notion de «travail forcé » généralement retenue par l'Organisation internationale du travail est, elle aussi, remise en question. Suivant la définition de l'OIT, le travail forcé ou obligatoire désigne « tout travail ou service exigé d'un individu sous la menace d'une peine quelconque et pour lequel ledit individu ne s'est pas offert de plein gré ${ }^{23}$. Or, pour Geneviève LeBaron et Nicola Phillips notamment ${ }^{24}$, cette définition est trop restrictive puisqu'elle occulte la contrainte d'ordre économique et par suite les formes réputées « volontaires » ou « contractuelles » de travail non libre.

Tom Brass défend, pour sa part, le concept de «travail non libre » dans son sens marxien pour deux raisons. La première est que ce concept opère une « rupture bienvenue » avec la notion de travail forcé, et son idée de menace, de contrainte ou de recours à la force (le "whips and chains " concept). Idée problématique pour Tom Brass en ce qu'elle conduit de facto à exclure nombre de formes de mise au travail de la définition même du problème et à envisager les formes d'exploitation selon une échelle du plus ou moins acceptable. Par opposition, Tom Brass propose de repartir de Marx pour qui deux critères sont requis pour distinguer des travailleurs

18 Alain Morice, "Comme des esclaves", ou les avatars de l'esclavage métaphorique ", Cahiers détudes africaines 179-180, n 3 (2005) : 1015-1036, à la p. 1029.

19 Roger Botte, «Les habits neufs de l'esclavage» (2005), à la p. 11.

20 Christophe Bormans, "Esclavage moderne et idéologie antique », Tiers-Monde 148, n 37 (1996) : 787-802, à la p. 788 .

21 Alain Morice, "Comme des esclaves ", ou les avatars de l'esclavage métaphorique " (2005), à la p. 1035.

22 Alain Morice et Bénédicte Michalon, « Les migrants dans l'agriculture : vers une crise de maind'oeuvre?", Études rurales 182, nº 2 (2008) : 9-28, à la p. 24.

23 OIT, Convention ( $\left.\mathrm{n}^{\circ} 29\right)$, Convention concernant le travail forcé ou obligatoire (Entrée en vigueur : 01 mai 1932) Genève, 14ème session CIT (28 juin 1930), art. 2.

24 Geneviève LeBaron et Nicola Phillips, "States and the Political Economy of Unfree Labour ", New Political Economy 1 (2018) : 1-21, à la p. 3. Voir aussi Todd Gordon, «Capitalism, Neoliberalism, and Unfree Labour ", Critical Sociology, (2018) : 1-19. 
libres : la privation des moyens de production d'une part, la liberté de pouvoir vendre personnellement sa force de travail d'autre part. Les travailleurs non libres sont ceux qui ne rencontrent pas ce second critère ${ }^{25}$.

La deuxième bonne raison de mobiliser ce concept de travail non libre tient à la théorie qu'il contient. Suivant sa définition marxienne, ce concept conserve l'idée que le travail non libre entretient un rapport dialectique avec le travail libre. Et c'est précisément ce rapport dialectique qui est au cœur de la thèse marxiste minoritaire de la déprolétarisation que soutient Tom Brass. Loin d'appartenir à la " préhistoire du capitalisme ", le travail non libre doit être compris comme "un enjeu crucial de la lutte des classes entre le capital et le travail ${ }^{26}$. Il permet $"$ aux producteurs capitalistes de dépolitiser, de discipliner et de rendre la main-d'œuvre moins chère ${ }^{27}$. Inversement, avec "léradication des relations de production non libres (...), il serait plus facile pour les travailleurs de s'unir, de s'organiser et de se battre en tant que prolétariat, au sens marxiste du terme ${ }^{28}$. Les mouvements de déprolétarisation du travail sont donc au centre des processus actuels de transformation du travail et du conflit de classes, "non entre une bourgeoisie naissante et des propriétaires féodaux, comme dans la thèse semi-féodale, mais entre le capital et sa main-d'œuvre passant d'une classe en soi à une classe pour soi ${ }^{29}$. Comme le résume Yann Moulier-Boutang, « [p]rolétarisation, déprolétarisation, re-prolétarisation apparaissent ainsi comme le premier enjeu constant de la lutte de classe ${ }^{30}$.

Cette thèse d'une relation dialectique entre liberté et non-liberté du travail dans le capitalisme trouve également un écho dans le champ juridique avec les travaux d'Alain Supiot. Il fait pour sa part l'hypothèse d'une « reféodalisation » par la voie de la contractualisation des rapports de travail. Ainsi, à l'encontre d'une vision linéaire des progrès juridiques, portée par le "positivisme moderne ", qui soutient que le contrat - la liberté contractuelle - a mis fin aux "statuts " hérités du féodalisme, Supiot insiste sur la permanence de régimes juridiques fondés sur "l'allégeance » et la " dépendance ", deux éléments caractéristiques du féodalisme ${ }^{31}$. Il invite les juristes à remettre en question la portée du libéralisme juridique et de la «contractualisation », entendue ici comme le " recul de l'hétéronomie face à l'autonomie » ${ }^{32}$. Car, de fait, c'est bien au nom de la liberté contractuelle, de la « loi pour soi », de la liberté de choisir ses propres "chaines " ${ }^{33}$, de la liberté d'être libre d'être non libre, que les travailleurs migrants et travailleuses migrantes

25

Tom Brass, «Modern Capitalism and Unfree Labor: The Unsaying of Marxism », Science \& Society $78, n^{\circ} 3$ (2014) : 288-311, à la p. 291 (notre traduction).

26 Tom Brass, «Unfree Labour as Primitive Accumulation? » (2011), à la p. 25 (notre traduction).

27 Ibid., à la p. 25 (notre traduction).

28 Tom Brass, «Modern Capitalism and Unfree Labor: The Unsaying of Marxism » (2014), à la p. 305 (notre traduction).

29 Tom Brass, « Free Markets, Unfree Labour: Old Questions Answered, New Answers Questioned », Journal of Contemporary Asia 45, $\mathrm{n}^{\circ} 3$ (2015) : 531-540.

30 Yann Moulier-Boutang, "Formes de travail non libre. "Accumulation primitive : préhistoire ou histoire continuée du capitalisme ?” " (2005), à la p. 1086.

31 Alain Supiot, " "Les deux visages de la contractualisation" : déconstruction du droit et renaissance féodale ", dans Approche critique de la contractualisation, (dir.) Sandrine Chassagnard-Pinet et David Hiez (Paris : LGDJ, 2007)19-44.

32 Ibid., à la p. 19.

33 Ibid., à la p. 29. 
temporaires se trouvent soumis à un droit du travail dérogatoire au droit commun. De ce point de vue, le contrat « librement consenti » participe de la déconstruction des acquis juridiques d'ordre public et constitue, en lui-même, un enjeu de lutte ${ }^{34}$.

À partir de ces quelques exemples, on voit bien en quoi les débats en cours viennent complexifier les critiques plus classiques de l'économie politique - qui réduisent l'exploitation capitaliste à la seule forme du salariat libre - et qu'ils sont l'occasion de mettre en cause une sociologie très " primitive qui suppose qu'il n'existe que trois sortes de statuts du travail : celui du salarié libre contractuel; celui du serf; celui de l'esclave et qui cherche à faire entrer tout le monde dans une de ces catégories ${ }^{35}$.

Reste que, au regard des avancées enregistrées par la sociologie féministe et intersectionnelle du travail, ces renouvellements théoriques restent insuffisamment critiques vis-à-vis de l'économie politique. Faute d'intégrer pleinement les divisions sexuelle et raciale du travail en tant que formes proprement sociales, historiques et politiques d'organisation du travail qui participent des processus en cours, ils en produisent de facto une lecture tronquée.

\subsection{Les apports des théories féministes matérialistes et intersectionnelles}

Par défaut, dans la mesure où elle contourne le problème des divisions sexuelles et raciales du travail non libre - qui en constituent pourtant des dimensions importantes -, la thèse de la déprolétarisation tend à laisser penser que le processus de production de ce travail non libre comme le conflit de classes dont il procède sont indépendants de la dynamique des rapports sociaux de sexe et de race. Pire, cette thèse peut laisser penser que les divisions sexuelle et raciale du travail ne relèvent pas de l'économie politique.

Les critiques féministes matérialistes et imbricationnistes ont pourtant amplement démontré en quoi cette exclusion des rapports de sexe et de race constitue un manquement au matérialisme qui vient fragiliser l'analyse de l'exploitation réellement existante et par conséquent celle des conditions de construction de solidarités. Par opposition, ces critiques ont réalisé des avancées décisives pour la compréhension de l'exploitation ${ }^{36}$.

Nous renvoyons d'abord ici le lecteur aux travaux pionniers de Christine Delphy qui sest notamment attaquée à la doxa marxiste qui veut que le capitalisme, à lui seul, constitue le tout de l'exploitation dans nos sociétés ${ }^{37}$. L'auteure a d'abord

34 Yann Moulier-Boutang, «Formes de travail non libre. “ Accumulation primitive : préhistoire ou histoire continuée du capitalisme?”"(2005), à la p. 1083.

35 Moses I. Finley, Économie et société en Grèce ancienne (Paris : La Découverte, 1984), 208 cité dans Christophe Bormans, «Esclavage moderne et idéologie antique » (1996), à la p. 788.

36 Voir en particulier Christine Delphy, L'ennemi principal. 1. Économie politique du patriarcat (Paris : Syllepse, 1998) : 31-56; Colette Guillaumin, «Pratiques du pouvoir et idée de Nature. (I) Lappropriation des femmes », Questions féministes 2 (1978a) : 5-30; Colette Guillaumin, "Pratiques du pouvoir et idée de Nature. (II) Le discours de la Nature ", Questions féministes 3 (1978b) : 5-20; Paola Tabet, La construction sociale de l'inégalité des sexes. Des outils et des corps (Paris : L'Harmattan "Bibliothèque du féminisme ", 1998).

37 Christine Delphy. "Un féminisme matérialiste est possible », Nouvelles Questions Féministes 4 (1982) : 50-86; Christine Delphy, « Pour une théorie générale de l’exploitation. En finir avec la théorie de la plus-value », Mouvements 26, n² 2 (2003) : 69-78; Christine Delphy, " Pour une théorie générale de l'exploitation ». Deuxième partie : repartir du bon pied (2004). 
montré comment ce dogme de la solitude du capitalisme opère selon une série de réductions et de confusions, en identifiant totalement plusieurs termes : léconomie et léconomie de marché, le travail et le travail salarié, l'exploitation et sa forme capitaliste. En aval, Christine Delphy a aussi mis en évidence l'une des principales implications de ce dogme, à savoir qu'il conduit à nier l'exploitation des femmes par les hommes comme celle des racisés par les non-racisés. « Au mieux le dogme permetil d'admettre qu'il existe des catégories de travailleurs plus désavantagés que les autres à l'intérieur du capitalisme ${ }^{38} "$, mais, d'une part, il ne permet pas d'expliquer ces désavantages sans recourir à l'idéalisme ou au naturalisme ${ }^{39}$ et, d'autre part, il nie qu'il existe des exploitations de type non capitaliste dans nos sociétés.

Par opposition, Christine Delphy plaide pour une théorie générale de l'exploitation et cette proposition fait écho aux théories imbricationnistes ${ }^{40}$ pour lesquelles les rapports sociaux de sexe, de race et de classe sont à la fois irréductibles les uns aux autres et constitutifs les uns des autres.

Nous référons ici à l'ensemble des analyses critiques qui sont maintenant des classiques du black feminism ${ }^{41}$, s'agissant de la théorisation de l'esclavage. À contrecourant de l'historiographie abolitionniste dominante faisant de «l'homme esclave de plantation » la figure paradigmatique de l'esclave, les théoriciennes féministes noires ont montré l'importance d'intégrer les expériences des femmes noires et de l'esclavage de maison dans la définition même de la catégorie d'esclavage. Nous renvoyons également le lecteur au travail plus récent de Patricia Hill Collins qui propose de retravailler léconomie politique du racisme à l'aune de la problématique du genre ${ }^{42}$ ainsi qu'aux analyses d'Evelyn Nakano Glenn qui montre comment la sociologie critique qui s'intéresse à la division raciale du travail aux États-Unis persiste à occulter le rapport particulier des femmes migrantes et racisées au travail, faute de tenir compte du travail domestique d'une part et de la division sexuelle du travail d'autre part ${ }^{43}$. Par opposition, Evelyn Nakano Glenn a démontré l'imbrication

38 Christine Delphy, «Pour une théorie générale de l'exploitation. En finir avec la théorie de la plus-value » (2003), à la p. 71.

39 Sur l'«Idée de Nature » telle qu'elle persiste à s'appliquer aux femmes et aux racisés, nous renvoyons tout particulièrement aux travaux de C. Guillaumin, L'idéologie raciste. Genèse et langage actuel (Paris : Mouton, 1972); Sexe, race et pratique du pouvoir : l'idée de nature (Paris : Côté-femmes, 1992).

40 Danièle Kergoat, Se battre disent-elles (Paris : La dispute, 2012); Patricia Hill Collins, Black Feminist Thought - Knowledge, Consciousness, and The Politics of Empowerment (Unwin Hyman: Boston, 1990); Patricia Hill Collins, " Gender, Black Feminism, and Black Political Economy ", Annals of the American Academy of Political and Social Science 568 (2000) : 41-53; Evelyn Nakano Glenn, "From Servitude to Service Work: Historical Continuities in the Racial Division of Paid Reproductive Labor ", Signs: Journal of Women in Culture \& Society 18 (1992) : 1-43; Evelyn Nakano Glenn, Forced to Care: Coercion and Caregiving in America (Harvard University Press: Cambridge, 2010); Rose Brewer. "Black Radical Theory and Practice: Gender, Race, and Class ", Socialism and Democracy $17, \mathrm{n}^{\circ} 1$ (2003) : 109-122.

41 Angela Davis, "Reflections on the Black Woman's Role in the Community of Slaves ", Black Scholar 3 (1971) : 81-100; Bonnie Thornton Dill, " The Dialectics of Black Womanhood »,) Signs 3, nº 4 (1979) : 543-555; Angela Davis, Women, Race and Class (New York: Vintage Books, 1981); bell hooks, Ain't I a Woman: Black Women and Feminism (Boston: South End Press, 1981); Patricia Hill Collins, Black Feminist Thought - Knowledge, Consciousness, and The Politics of Empowerment (1990).

42 Patricia Hill Collins, « Gender, Black Feminism, and Black Political Economy » (2000).

43 Evelyn Nakano Glenn, "From Servitude to Service Work: Historical Continuities in the Racial Division of Paid Reproductive Labor» (1992). 
dynamique des divisions sexuelles et raciales du travail, leur continuité historique avec l'esclavage et l'insuffisance des luttes pour l'émancipation qui ne voient pas ces imbrications ${ }^{44}$.

Dans la lignée de ces travaux, c'est plus restrictivement ici sur l'organisation sexuée du travail migrant temporaire que nous proposons de centrer l'analyse, en suivant l'une des propositions clés du féminisme matérialiste. Il sagit en particulier de renverser l'ordre dans lequel l'idéologie naturaliste envisage le rapport entre catégories de sexe et division du travail. Suivant cette idéologie, l'existence d'hommes et de femmes précède l'organisation du travail. Par opposition, la division sexuelle du travail est ici appréhendée, de manière matérialiste, en tant quelle est constitutive d'un clivage de sexe au sein même du travail non libre : ce par quoi se produisent des travailleurs non libres et des travailleuses non libres. Tenir compte de cette sexuation dans et par le travail et de la manière dont elle peut structurer les conflits du travail suppose alors d'examiner les modalités de mise au travail des unes et des autres et ce qu'elles impliquent.

\section{La division sexuelle du travail migrant temporaire canadien et ses implications}

En 2015, les deux secteurs de l'agriculture primaire et du travail domestique résident regroupaient, à eux seuls, plus de $58 \%$ de l'ensemble des 90000 travailleurs et travailleuses admis au Canada, en vertu du Programme des travailleurs étrangers temporaires ${ }^{45}$. Comme nous le verrons, ces deux secteurs sont fortement sexués (2.1) et cette ségrégation produit des expériences fortement différenciées du travail non libre que nous allons tenter d'illustrer, en insistant sur deux enjeux en particulier : les espaces et relations de travail (2.2) d'une part, les rapports au temps travaillé d'autre part (2.3).

\subsection{Le travail domestique et le travail agricole : deux secteurs d'emplois fortement sexués}

En 2013, selon l'OIT, on dénombrait 150 millions de travailleurs migrants et travailleuses migrantes (temporaires ou non) dans le monde : 83,7 millions d'hommes $(55,7 \%)$ et 66,6 millions de femmes (44,3\%). D’après les données disponibles, hommes et femmes étaient alors représentés dans une proportion « presque identique » dans le secteur du travail agricole ${ }^{46}$, tandis que celui du travail domestique était fortement féminisé : les femmes y représentaient 73,4\% de la main-d’œuvre.

44 Y compris celle des luttes féministes qui restent fondées sur la seule expérience des femmes blanches et bien classées de l'exploitation.

45 Canada, Chambre des Communes, Programmes des travailleurs étrangers temporaires, $42^{\mathrm{e}}$ législature, 2016. <https://www.noscommunes.ca/Content/Committee/421/HUMA/Reports/RP8374415/ humarp04/humarp04-f.pdf>; Canada, Emploi et développement social Canada, Le plan décrivant la marche à suivre pour le Programme des travailleurs étrangers temporaires et le Programme de mobilité internationale, 2017. <https://www.canada.ca/fr/emploi-developpement-social/services/ travailleurs-etrangers/rapports/plan.html>.

46 OIT, Migration de main-d'ouvre - Nouvelle donne et enjeux de gouvernance - Rapport IV (Genève, Publications de l'OIT, 2017), para 18. <http://www.ilo.org/wcmsp5/groups/public/---ed_norm/--relconf/documents/meetingdocument/wcms_550363.pdf $>$. 
Cette distribution sexuée du travail migrant varie cependant non seulement d'une région à l'autre mais aussi selon les pays.

Au regard de ces variations, à la différence d’autres programmes, les programmes canadiens d'immigration de travail temporaire semblent opérer une ségrégation quasi systématique du marché du travail non libre entre secteur d’emploi féminin et secteur d'emploi masculin, travaux d'hommes et travaux de femmes ${ }^{47}: 97 \%$ des 45000 travailleurs migrants temporaires ${ }^{48}$ employés dans le secteur agricole sont des hommes ${ }^{49}$; souvent recrutés parmi les populations autochtones, ils sont mexicains ou guatémaltèques dans l'immense majorité des cas. Par ailleurs, 95,5\% des 13000 travailleuses domestiques employées dans le cadre du Programme des « aides familiaux » sont des femmes, venues des Philippines dans plus de $90 \%$ des cas ${ }^{50}$. Les deux secteurs sont donc fortement ethnicisés mais aussi sexués ${ }^{51}$ (féminisés et masculinisés), et ce, historiquement. La domesticité s'est plus précisément féminisée au cours du XIX ${ }^{\mathrm{e}}$ siècle au Canada et les femmes nont été autorisées à participer au Programme des travailleurs agricoles saisonniers qu'un quart de siècle après sa mise sur pied, soit en $1989^{52}$.

Cette ségrégation par secteurs d'activité contribue à expliquer la manière dont la réflexion sur le travail non libre s'organise dans le contexte canadien. En somme, il n'est jamais question des hommes dans les recherches qui s'intéressent au travail domestique migrant ${ }^{53}$ et il est rarement question des femmes au sujet du travail agricole saisonnier. Les recherches qui font exception sont cependant particulièrement instructives. Jenna L. Hennebry qui s'intéresse non pas aux travailleuses migrantes mais aux femmes de travailleurs agricoles saisonniers restées au pays montre le surtravail qui leur revient en l'absence de leurs conjoints ${ }^{54}$. De son côté, Marie-France Labrecque met en évidence le processus de sélection par lequel les femmes mayas du Yucatan se trouvent exclues du travail agricole saisonnier ${ }^{55}$.

47 Canada, Statistique Canada, Les ressortissants étrangers qui travaillent temporairement au Canada (2014). <https://www.statcan.gc.ca/pub/11-008-x/2010002/article/11166-fra.htm>.

48 Canada, Chambre des Communes (2016).

49 Kerry Preibisch et Evelyn Encalada, «The other side of 'El Otro Lado': Mexican migrant women and labor flexibility in Canadian agriculture ", Signs: Special issue on Women in Agriculture 35, $\mathrm{n}^{\circ} 2(2010): 289-316$.

50 Sur le dispositif d'exportation de main-d'œuvre de l'État philippin, nous renvoyons en particulier à Julien Debonneville, «Les écoles du care aux Philippines. Le devenir travailleuse domestique au prisme de l’altérité », Revue Tiers Monde 1, nº 217 (2014) : 61-78. Voir également Rhacel Salazar Parrenas, Servants of Globalization: Women, Migration and Domestic Work, $2^{\text {nd }}$ ed. (Stanford: Stanford University Press, 2015).

51 À noter que le processus de recrutement de la main-d'œuvre agricole qui permet aux employeurs de sélectionner les travailleurs sur la base de leur sexe et de leur nationalité a été dénoncé comme discriminatoire par la Commission ontarienne des droits humains. Canada, Commission Ontarienne des droits de la personne, Énoncé de position - Discrimination sexuelle en matière de recrutement dans le cadre du Programme des travailleurs agricoles saisonniers, 2014. <http://www.ohrc.on.ca/en/ news_centre/position-statement---discrimination-basis-sex-recruitment-seasonal-agriculturalworkers-program>.

52 Pauline Gardiner Barber, « The Ideal Immigrant? Gendered class subjects in Philippine-Canada migration », Third World Quaterly 29 (2008) : 1265-1285.

53 Sur le travail domestique masculin en France et en Italie, voir Francesca Scrinzi, Genre, migrations et emplois domestiques en France et en Italie : construction de la non-qualification et de l'altérité ethnique (Paris : Petra, 2013).

54 Jenna L. Hennebry, "Transnational Precarity: Women's Migration Work and Mexican Seasonal Agricultural Migration ", International Journal of Sociology 44, $n^{\circ} 3$ (2014) : 42-59.

55 Marie-France Labrecque, La migration saisonnière des Mayas du Yucatan au Canada : la dialectique de la mobilité (Québec : Presses de l’Université Laval, 2016). 
Enfin, la recherche menée par Kerry Preibisch et Evelyn Encalada éclaire les modalités spécifiques de mise au travail des rares femmes migrantes qui sont effectivement recrutées dans le secteur du travail agricole : affectées à certaines productions (fruits, horticulture ou floriculture) et à des tâches séparées (emballer, planter les semis ou couper des fleurs), elles sont aussi soumises à un contrôle spécifique de la part des employeurs (absence de mobilité, harcèlement ${ }^{56}$, etc. $)^{57}$.

Par ailleurs, toujours dans le contexte canadien, les deux programmes et les deux formes de travail non libre qu'ils produisent sont le plus souvent étudiés séparément, y compris dans les recherches qui intègrent la problématique du genre ${ }^{58}$, si bien que lorganisation sexuée du travail migrant temporaire est en elle-même, comme dans ses effets, encore peu étudiée.

\subsection{Le travail agricole et le travail domestique : les espaces et les relations de travail}

Le travail migrant temporaire est organisé suivant une série de séparations qui structurent historiquement l'organisation du travail : privé/public, intérieur/extérieur, travaux lourds/travaux présumés légers, travail de la terre/domestique et/ou de soin. Selon ces séparations, les corps se trouvent affectés à des tâches comme à des espaces de travail distincts. Ici, après avoir précisé le contenu des activités de travail, nous voudrions insister sur les espaces du travail et sur ce qu'ils impliquent comme relations de travail.

\subsubsection{Les activités de travail}

Les ouvriers agricoles sont affectés au travail de la terre, tandis que les aides familiales le sont au «travail d'entretien matériel d'autrui » pour reprendre la formulation de Colette Guillaumin. Dans les deux cas, les corps sont fortement sollicités par l'activité de travail, mais ils sont affectés à des usages séparés qui rappellent la distinction mise en évidence par Colette Guillaumin, en 1978, entre les rapports d'appropriation dans lesquels les corps sont réduits « à l'état de chose, d'outil dont l'instrumentalité est appliquée (ou applicable) à d'autres choses (agricoles, mécaniques, animales...) » et ceux qui concernent « la réduction

56 Sur les violences sexuelles subies par les travailleuses agricoles, on renverra au jugement rendu en 2015 par le Tribunal des droits de la personne de l'Ontario condamnant deux employeurs à des « dommages records ». O.P.T. v. Presteve Foods Ltd., 2015 HRTO 675 (CanLII).

57 Kerry Preibisch et Evelyn Encalada, « The other side of 'El Otro Lado': Mexican migrant women and labor flexibility in Canadian agriculture " (2010), à la p. 302. Voir également Barbara Pini et Belinda Leach (dir.), Reshaping Gender and Class in Rural Spaces (London: Routledge, 2013); Melissa Paciulan et Kerry Preibisch, "Navigating the Productive/Reproductive Split: Latin American Transnational Mothers and Fathers in Canada's Temporary Migration Programs ", Transnational Social Review 3, $\mathrm{n}^{\circ} 2$ (2013) : 173-192.

58 Kendra Strauss et Siobhàn McGrath, « Temporary migration, precarious employment and unfree labour relations: Exploring the 'continuum of exploitation' in Canada's Temporary Foreign Worker Program ", Geoforum 89 (2017) : 199-208; Nandita Sharma, " On being not Canadian: The social organization of "migrant workers" in Canada ", Canadian Review of Sociology 38, ${ }^{\circ} 4$ (2001) : 415-439; Sylvia Fuller, Leah F. Vosko, " Temporary employment and social inequality in Canada: Exploring intersections of gender, race and immigration status ", Social indicators research 88, $n^{\circ} 1$ (2008) : 31-50; Daiva Stasiulis, "Canada: Gender and Migration », in The Encyclopedia of Global Human Migration (2013). 
à l'état d'outil dont l'instrumentalité s'applique de surcroît et fondamentalement à d'autres humains ${ }^{59}$.

Ni la réglementation ni le contrat de travail ne prévoient de description de tâches dans le cas des ouvriers agricoles. La grande majorité de ceux que nous avons rencontrés sont embauchés dans des fermes maraîchères ou horticoles qui produisent des légumes (89/92). Ils travaillent principalement à l'extérieur ou dans des serres, «beau temps, mauvais temps », pour reprendre l'expression d'un employeur ${ }^{60}$. Ils cultivent en moyenne deux ou trois produits, le plus souvent des légumes, et accomplissent, pour ce faire, des tâches très variées : désherber les champs; déplacer les pierres; planter les semis; labourer et sarcler la terre; récolter les produits; trier les légumes; les nettoyer; empaqueter les produits à la main ou à la machine; charger les camions; tondre le gazon, etc. Couteaux, tracteurs, autres machines agricoles et produits chimiques sont les outils les plus couramment utilisés. Quand le travail vient à manquer, il peut arriver qu'ils soient affectés à d'autres travaux considérés comme lourds : repeindre ou rénover des bâtiments, réparer des silos, etc.

Les ouvriers exécutent un travail qu'ils qualifient de " physique », " extrêmement usant » et sont soumis à une cadence excessive. Force et endurance semblent tout particulièrement requises, notamment pour la récolte de certains produits - quand les travailleurs sont allongés à plat ventre sur des planches en bois tirées par un tracteur pour ramasser les concombres par exemple - ou pour l'empaquetage ${ }^{61}$. Ils décrivent aussi un travail dangereux où les corps sont souvent blessés : coupures, jambes cassées, arthrite, intoxications aux produits chimiques, maux de dos sont récurrents. Les accidents sont fréquents et souvent liés aux machines qui sont lourdes et tranchantes.

Au moment d'expliquer la pénibilité du travail, les ouvriers évoquent la lourdeur des tâches, la dureté du climat, les injonctions constantes des contremaîtres à travailler plus vite, un travail sous pression et des relations de travail particulièrement paternalistes ${ }^{62}$. Des insultes racistes aux violences physiques en passant par les promesses de bon (ou mauvais) traitement et les déductions de paie, ce sont les menaces d'exclusion du programme qui semblent surdéterminer le rapport au travail. Voici quelques commentaires recueillis :

«Le mieux, c'est de ne pas parler. » «Si un travailleur dit quelque chose, s'il revendique ses droits, il ne revient pas l'année suivante. Ils nous traitent mal, très mal, et on doit le supporter. » «Oui. Pour un petit problème, ils t'envoient au Mexique. »

À la différence des travailleurs agricoles, les travailleuses domestiques sont affectées au travail de soin à demeure où le « rapport de travail sétablit principalement

59 Colette Guillaumin, « Pratiques du pouvoir et idée de Nature. (I) L'appropriation des femmes » (1978), à la p. 16.

60 FERME, Mémoire de FERME à la Commission de consultation sur les pratiques d'accommodements reliées aux différences culturelles, René Mantha, Québec, FERME (2007), à la p. 12.

61 Pour une illustration des conditions de travail, sanctionnées par le tribunal administratif du travail, nous renvoyons à l’affaire Orantes Silva et 9009-1729 Québec inc, 2016 QCTAT 2155 (CanLII).

62 Voir Jorge Pantaleón et Lucio Castracani, « Travail, morale et dépendance personnelle - les ouvriers agricoles mexicains et guatémaltèques dans les fermes québécoises ", Anthropologie et Sociétés 41, $\mathrm{n}^{\circ} 1$ (2017) : 91-106; Danièle Bélanger et Guillermo Candiz, "Fraises douces amères : territoire et précarité chez les travailleurs agricoles migrants de la région de Québec », Cahiers de géographie du Québec 59 (2015) : 7-28. 
entre deux femmes ${ }^{63}$. Théoriquement, les patrons et patronnes sont tenues de décrire " les responsabilités et les tâches liées aux soins » de l'aide familiale dans son contrat de travail. Sur ce point, les services d'immigration précisent : «Les travaux domestiques, le ménage et les autres tâches ménagères semblables, comme la préparation des repas, sont permis dans une faible proportion de l'ensemble des tâches, et lorsqu'elles sont clairement liées aux tâches de soins à la ou aux personnes, elles ne peuvent toutefois pas être la tâche principale " ${ }^{64}$. Comme dans le cas du travail agricole, celui de l'aide familiale est donc peu défini. La distinction opérée entre tâches ménagères et tâches de soin n'est par ailleurs pas opératoire en pratique pour les travailleuses que nous avons rencontrées. La liste des tâches répertoriées au moment de décrire leur travail témoigne non seulement du fait que ces tâches sont étroitement imbriquées mais aussi du caractère indéfini du travail d'aide familiale : lever, faire la toilette de la personne âgée, administrer les médicaments, habiller, préparer le déjeuner, surveiller, amener les enfants à l'école, laver le linge, le repasser, le ranger, préparer les bagages, planifier les repas, les courses, faire l'épicerie, nettoyer la cuisine, remplir le lave-vaisselle, le vider, débarrasser, récurer la ou les salles de bain, passer la balayeuse, nettoyer le garage, laver la voiture, soccuper de la piscine, promener les animaux, les faire manger, jardiner, jouer avec les enfants, préparer la collation, le souper et le café, coucher les enfants...

Comme le résume une travailleuse à qui l'on a demandé de décrire son travail :

«Superwomen. You can put Superwoman. I can be mom, daddy, whatever.

Cleaning, cooking, meal-planning, pet care, do the groceries, spend time with the children ».

Toutes, par ailleurs, ont souligné l'importance des tâches ménagères dans ce travail d'aide familiale. Parmi les tâches qui leur incombent, plusieurs imposent des gestes à effectuer directement sur les corps, élément qui constitue ainsi une dimension essentielle de leur activité. Ce travail implique par ailleurs une "posture » discrète d'attention à autrui, visant à anticiper et à ajuster la tâche aux besoins. Comme cela est abondamment documenté, " le travail domestique, on le sait, pour être bien fait ne doit pas se voir et ne doit pas gêner la vie quotidienne de qui en bénéficie, sinon, il est raté. $[\ldots]{ }^{65}$. Il s'agit d'un travail monotone et répétitif, qui s'effectue sous contrainte temporelle, rythmé par les horaires scolaires comme par ceux des soins corporels, et marqué par l'invisibilité.

\subsubsection{Les espaces et les relations de travail}

D’un côté comme de l'autre, les travailleurs et travailleuses temporaires sont attachés à un employeur/une employeuse unique et il s'agit là de l'un des éléments

63 Blandine Destremau et Bruno Lautier, « Introduction : Femmes en domesticité. Les domestiques du Sud, au Nord et au Sud », Tiers-Monde 43, n 170 (2002) : 249-264, à la p. 253. Sur ce rapport servantes/madames, nous renvoyons au travail pionnier de Judith Rollins, «Entre femmes [Les domestiques et leurs patronnes] ", Actes de la recherche en sciences sociales 84 (1990) : 63-77.

64 Canada, Citoyenneté et immigration Canada, IP 04 - Traitement des aides familiaux résidants au Canada, Immigration Canada (5 novembre 2015), à la p. 9. <http://www.cic.gc.ca/francais/ ressources/guides/ip/ip04-fra.pdf>.

65 Pascale Molinier, «Des féministes et de leurs femmes de ménage : entre réciprocité du care et souhait de dépersonnalisation », Multitudes 37-38, n² 2 (2009) : 113-121, à la p. 116. 
structurants du travail non libre. Dans les deux cas, l'impossibilité de changer d'employeur/d'employeuse, doublée de la crainte du renvoi et de l'expulsion du territoire structure les rapports au travail. Ce que ces travailleurs et ces travailleuses disent des relations concrètes de travail témoignent de rapports de dépendance personnelle avec les employeurs/employeuses, mais ceux-ci se jouent différemment en raison des espaces de travail.

La très grande majorité des travailleurs agricoles rencontrés au cours de l'enquête travaillaient dans des fermes qui embauchaient plus de 10 employés (80\%) et fréquemment plus de 50 employés (25\%). À la différence des travailleuses domestiques qui sont isolées les unes des autres, dispersées dans les familles pour lesquelles elles travaillent et pour qui le rapport au travail est à la fois personnalisé et individualisé, les travailleurs agricoles saisonniers sont ségrégués et confinés dans un espace-temps de facto commun ${ }^{66}$.

La journée est la même : ils la commencent et la finissent en même temps, après quoi ils regagnent aussi collectivement leur logement. Dans leur cas, il sagit d'une vie en collectivité imposée. Ces logements sont souvent surpeuplés, insalubres; on y trouve de la vermine, il y fait particulièrement chaud ou froid selon les saisons. Il est fréquemment interdit aux travailleurs de recevoir de la visite et ils sont parfois surveillés par des caméras vidéo. Les travailleurs dénoncent une constante promiscuité à laquelle ils ne peuvent que difficilement échapper compte tenu de l'absence de transport facilement accessible qui tend à les immobiliser. Pas plus que les travailleuses domestiques, ils ne sont donc chez eux.

Pour elles, comme l'ont montré F.-X. Devetter et D. Messaoudi, « le lieu du travail salarié se confond avec l'espace privé » de celles et ceux qui les emploient et il s'agit là d'une caractéristique qui implique « l'éclatement du collectif de travail ». Les aides familiales sont donc « très largement non concernées par un temps de travail collectif qui marque souvent les autres professions considérées comme peu ou non qualifiées ${ }^{67}$.

De surcroît, la relation de domesticité se caractérise par la proximité physique et la "domination rapprochée " ${ }^{68}$ qu'elle permet, laquelle a partie liée avec cet espace spécifique de travail que constitue le domus. Les travailleuses doivent en effet composer dans cet espace privé des employeurs/employeuses qu'elles sont tenues de respecter comme tel, ce qui implique pour elles de maintenir une juste distance. Le travail exige discrétion, invisibilité et déférence, d'autant que ce qui est capté ou extorqué à travers cette relation servante-servi/servie ${ }^{69}$, c'est aussi un statut : le fait de faire faire et de ne pas faire soi-même ce travail constitue, aujourd'hui comme hier, une marque distinctive pour les employeurs/employeuses. Le travail des unes

66 Seuls $16 \%$ des répondants avaient une chambre individuelle.

67 François-Xavier Devetter et Djamel Messaoudi, « Les aides à domicile entre flexibilité et incomplétude du rapport salarial : conséquences sur le temps de travail et les conditions d'emploi ", La Revue de l'Ires 3, nº 78 (2013) : 51-76.

68 Sur ce concept de "domination rapprochée ", voir Dominique Memmi, " Aides à domicile et domination rapprochée ", La Vie des idées (2016). <http://www.laviedesidees.fr/Aides-a-domicile-etdomination-rapprochee.html>; ce cadre de la famille est d'ailleurs propice aux pratiques de harcèlement, y compris sexuel : PN v. FR and another (No. 2), 2015 BCHRT 60 (CanLII).

Colette Le Petitcorps, "Travailler chez les autres ", La nouvelle revue du travail - en ligne 9 (2016). 
est la condition du statut des autres : comme cela est abondamment démontré, le service domestique constitue l'une des formes paradigmatiques des rapports sociaux de classe et de race entre femmes.

Alors qu'une proximité physique est constamment imposée aux travailleurs agricoles qui vivent en collectivité, dans un entre-soi masculin qui leur est imposé, les aides familiales, elles, sont de facto séparées les unes des autres et prises dans une présence constante avec celles et ceux pour lesquels elles travaillent.

\subsection{Le rapport au temps travaillé}

Cette assignation différenciée au travail ne se traduit pas seulement par des rapports distincts aux espaces et aux corps mais également par des rapports dissymétriques au temps travaillé payé et non payé.

La grande majorité des travailleurs agricoles arrive au printemps et quitte le pays à l'automne, en octobre ou en novembre. Ce travail n'a rien de temporaire, si ce n'est son caractère saisonnier. Au moment des entretiens, les travailleurs avaient déjà participé 9 ou 10 fois au programme.

À leur départ du Mexique ou du Guatemala, ils ne connaissent ni le nombre d'heures pour lesquelles ils seront embauchés ni la date à laquelle prendra fin leur contrat. Les employeurs décident de la durée journalière du travail et peuvent mettre fin au contrat avec une semaine de préavis seulement. Dans les faits, la grande majorité des ouvriers agricoles font une saison de 4 à 6 mois. Ils disent travailler en moyenne 10 heures par jour, 6 jours sur 7. Le nombre d'heures travaillées par jour varie cependant fortement d'un travailleur à l'autre et aussi pour un même travailleur au cours de la saison. La journée peut être de 14 heures au mois de juillet et de 6 heures en avril, tandis que les semaines de pluie peuvent être non travaillées. Le salaire net moyen est de 522,12 \$ par semaine avec des écarts qui vont de 250 $\$$ par semaine à $875 \$$ (pour 14 heures par jour sur 6 jours, soit 84 heures).

Dans tous les cas, la plupart des travailleurs ne se plaignent pas tant des longues heures de travail que de l'incertitude et de l'absence de travail, le cas échéant. Ils sont là pour « faire des heures » comme l'explique l'un d'eux. Dans leur cas, la journée de travail, si longue soit-elle, commence et se termine, tandis que les heures supplémentaires sont le plus souvent comptées et payées, sans être cependant majorées. Quand ce n'est pas le cas, c'est par ailleurs le seul droit qu'ils osent revendiquer.

En somme, le temps travaillé n'est évoqué comme un enjeu de conflit ou de revendication que lorsque le travail vient à manquer. Ce constat rejoint ceux d'autres enquêtes menées parmi des travailleurs agricoles saisonniers ${ }^{70}$. Il faut dire que la faiblesse du salaire horaire (10,75 \$) et l'absence de protection contre le chômage contraignent les travailleurs à faire le maximum d'heures pour rembourser leurs dettes (58/93 se sont endettés pour venir), " rentabiliser » leur participation au programme et espérer compenser financièrement le coût que représente léloignement de leurs familles. Or, ils sont concrètement confrontés à de longues

70 Danièle Bélanger et Guillermo Candiz, « Fraises douces amères : territoire et précarité chez les travailleurs agricoles migrants de la région de Québec » (2015), à la p. 16. 
périodes d'inactivité où ils sont contraints de rester sur place et de payer un loyer pour certains.

Dans le cas des aides familiales en revanche, le caractère illimité du temps réellement travaillé constitue un objet central de conflit et de revendication.

«Quand tu vis dehors, c'est très bien, tu as du temps, tu commences et tu finis ton travail. Mais quand tu vis dedans, tu travailles aussi longtemps qu'ils et elles le veulent. »

«Tu ne peux rien faire de personnel pendant la semaine... Aucune intimité (approbation du groupe)» (extraits de la discussion du deuxième atelier).

La grande majorité de celles que nous avons rencontrées travaillaient 5 jours par semaine (24/33) et quittaient la maison de leurs employeurs les fins de semaine, le vendredi ou le samedi. Plusieurs (6) travaillaient cependant 6 jours par semaine, et 3, tous les jours. Elles déclaraient majoritairement (26/33) des journées de 9 heures; 7 d'entre elles, des journées de plus de 14 heures, payées 40 heures la semaine. Toutes insistaient en outre sur la difficulté de mesurer le temps d'activité et relevaient l'absence de temps hors travail : « Ce n'est pas vraiment que tu travailles $24 \mathrm{~h} / 24 \mathrm{~h}$; c'est... n'importe quand, dès qu'ils ont besoin de toi. » « ... [m] on employeure, parce qu'elle est paralysée, elle a absolument besoin d'aide $24 \mathrm{~h} / 24 \mathrm{~h}$, sept jours sur sept. Toujours. Moi, comme aide familiale, je n'ai pas un jour de pause. »

La résidence implique ainsi une mise à disposition permanente où le temps de pause se confond toujours - et à la différence des travailleurs agricoles - avec le temps travaillé. Comme le résume une répondante : «... [p] endant mes 5 jours de travail, je n'ai pas de vie ». Il est fréquent que les employeurs/employeuses viennent cogner aux portes de chambre des employées pour leur assigner une tâche additionnelle, à n'importe quelle heure. Certains employeurs/certaines employeuses autorisent même leurs enfants à rester dans les chambres des travailleuses en soirée. Une employeuse exige de son employée qu'elle laisse sa porte ouverte en permanence pour pouvoir veiller sur sa mère. D’autre part, les travailleuses sautorisent peu de sorties du domicile des employeurs : certaines doivent demander la permission avant de quitter la résidence, d'autres nont pas les clés de leur logement. Une travailleuse a déclaré à ce sujet : «... [j] e suis devenue un chien attaché à mon employeur. »

De plus, contrairement aux travailleurs agricoles, toutes les travailleuses affirmaient avoir fait des heures "supplémentaires " ni payées ni même réclamées. Dans ce travail, " c'est comme ça ». " Pourquoi irais-tu demander à ton employeur; Pourquoi ferais-tu ça? Tu as 24 mois pour finir alors $90 \%$ de ton esprit te dit «ne fais pas ça ", tu risques de te faire virer. Alors, tu ne fais rien. Même moi qui connais mes droits. Et tu sais, les employeurs, ils connaissent bien ta situation alors même s'ils ont besoin de toi, ils vont te menacer de te licencier si tu ne fais pas comme ils veulent. » On voit bien ici que les mesures du travail prévues au contrat des aides familiales sont inexistantes dans les faits ${ }^{71}$.

Finalement, les travailleuses que nous avons rencontrées recevaient entre 330 \$ et 400 \$ par semaine. Au regard de la moyenne du temps travaillé qu'elles

71 Elsa Galerand et Martin Gallié, « L’obligation de résidence : un dispositif juridique au service d’une forme de travail non libre », Revue Interventions économiques 51- en ligne (2014) : 1-26. 
déclaraient au moment de nos échanges, c'est-à-dire sans compter leurs heures de disponibilité, leur salaire horaire moyen était de 6 \$.

Dans les deux cas, l'obligation de vivre au travail peut se lire comme l'un des moyens d’organiser de manière optimale la disponibilité des travailleurs et des travailleuses. De facto, la résidence assure leur confinement et, par là, leur exposition permanente au travail à faire selon les besoins. Mais l'espace privé du travail domestique, la relation de travail qui s'y noue avec sa proximité physique construit un rapport particulier au temps : alors que le temps du travail agricole est régulé par les saisons et la capacité de travail de la main-d'œuvre, le travail domestique dépend des « besoins horaires d'autrui " ${ }^{72}$, du temps nécessaire à en "prendre soin », du moment où les employeurs/employeuses ont faim, sont fatigués/fatiguées. Il y a donc bien une particularité du travail d'entretien d'autrui dans la mesure où il induit pour celles qui y sont affectées une relation directe et personnalisée dans laquelle une disponibilité permanente est requise, d'où l'importance de ces heures de présence qui ne sont jamais comptées.

Finalement, si une partie des tâches des aides familiales est prescrite dans le contrat de travail, ce qui les fait entrer dans le circuit salarial et dans la catégorie de la production que l'on peut mesurer, on voit bien qu'une autre partie, dont celle que l'on peut désigner en termes de charge mentale à la suite de Monique Haicault, y échappe ${ }^{73}$. A contrario, les tâches des travailleurs agricoles sont toujours nommées et comptabilisées; toutes les heures de travail, ou presque, sont payées.

\section{Conclusion}

Dans la mesure où elle fait l'impasse sur le problème des divisions sexuelles et raciales du travail, en tant que formes proprement sociales d'organisation du travail, la thèse de la déprolétarisation laisse penser que le travail non libre comme le conflit de classes - dont il procède et auquel il participe - sont indépendants de la dynamique des rapports sociaux de sexe et de race.

Par opposition, les critiques féministes matérialistes et imbricationnistes de l'économie politique nous invitent à faire l'hypothèse d'une recomposition des rapports de sexe, de race et de classe en tant que régimes spécifiques d'exploitation et d'appropriation du travail qui entretiennent des liens de coconstruction et qui structurent, ensemble, les réorganisations du travail. Résolument matérialistes, ces analyses nous invitent tout particulièrement à renverser l'idéologie naturaliste selon laquelle l'existence d'hommes et de femmes, de racisés/racisées et de nonracisés/-racisées, précède et explique les divisions sexuelle et raciale du travail. A contrario, ces formes sociales - et non pas naturelles - de division du travail doivent être non seulement remises en question mais appréhendées en tant qu’elles sont constitutives de clivages : ce par quoi se produisent des fractions ou des segmentations au sein même du travail.

72 Danielle Chabaud-Rychter, Dominique Fougeyrollas-Schwebel et Françoise Sonthonnax, Espace et temps du travail domestique (Paris : Méridiens, 1985).

73 Monique Haicault, "La gestion ordinaire de la vie en deux », Sociologie du Travail 26, n 3 (1984) : 268-277. 
Tenir compte de ces séparations dans et par le travail est alors indispensable pour la compréhension des conflits du travail et cela suppose d'examiner les modalités de mise au travail des unes et des autres et ce quelles impliquent.

C'est dans cette perspective que nous avons tenté de rendre compte de la sexuation du travail migrant temporaire au Canada et de ses effets en ce qui concerne les rapports au travail, aux espaces de travail et au temps travaillé. Au regard de nos premiers résultats, il nous semble que les conditions et les possibilités d’organisation collective des travailleurs migrants et des travailleuses migrantes sont bel et bien structurées par les rapports sociaux de sexe. Non seulement les enjeux de lutte (les revendications quant au temps de travail par exemple) mais aussi les modalités de passage au collectif des unes et des autres paraissent différenciés tant en raison des formes distinctes de mesure du travail agricole et domestique quen raison des espaces et des relations de travail. À la vie en collectivité imposée aux uns soppose l'isolement des autres. Faute de tenir compte de ces clivages internes, les tentatives de construction de solidarités entre travailleurs et travailleuses étrangers temporaires pourraient bien se trouver fragilisées.

Elsa Galerand

Professeure, département de sociologie

Université du Québec à Montréal, Montréal (Québec)

galerand.elsa@uqam.ca

Martin Gallié

Professeur, département des sciences juridiques

Université du Québec à Montréal, Montréal (Québec)

gallie.martin@uqam.ca 